\title{
Towards Task-Oriented Dialogue in Mixed Domains
}

\author{
Tho Luong Chi \\ FPT Technology Research Institute \\ FPT University, Hanoi, Vietnam \\ tholc2@fpt.com.vn
}

\author{
Phuong Le-Hong \\ FPT Technology Research Institute \\ Vietnam National University, Hanoi, Vietnam \\ phuonglh@vnu.edu.vn
}

\begin{abstract}
This work investigates the task-oriented dialogue problem in mixed-domain settings. We study the effect of alternating between different domains in sequences of dialogue turns using two related state-of-the-art dialogue systems. We first show that a specialized state tracking component in multiple domains plays an important role and gives better results than an endto-end task-oriented dialogue system. We then propose a hybrid system which is able to improve the belief tracking accuracy of about $28 \%$ of average absolute point on a standard multidomain dialogue dataset. These experimental results give some useful insights for improving our commercial chatbot platform FPT.AI, which is currently deployed for many practical chatbot applications.
\end{abstract}

Index Terms-task-oriented dialogue; multi-domain belief tracking; mixed-domain belief tracking; natural language processing

\section{INTRODUCTION}

In this work, we investigate the problem of task-oriented dialogue in mixed-domain settings. Our work is related to two lines of research in Spoken Dialogue System (SDS), namely task-oriented dialogue system and multi-domain dialogue system. We briefly review the recent literature related to these topics as follows.

Task-oriented dialogue systems are computer programs which can assist users to complete tasks in specific domains by understanding user requests and generating appropriate responses within several dialogue turns. Such systems are useful in domain-specific chatbot applications which help users find a restaurant or book a hotel. Conventional approach for building a task-oriented dialogue system is concerned with building a quite complex pipeline of many connected components. These components are usually independently developed which include at least four crucial modules: a natural language understanding module, a dialogue state tracking module, a dialogue policy learning module, and a answer generation module. Since these systems components are usually trained independently, their optimization targets may not fully align with the overall system evaluation criteria [1]. In addition, such a pipeline system often suffers from error propagation where error made by upstream modules are accumuated and got amplified to the downstream ones.

To overcome the above limitations of pipeline task-oriented dialogue systems, much research has focused recently in designing end-to-end learning systems with neural networkbased models. One key property of task-oriented dialogue model is that it is required to reason and plan over multiple di- alogue turns by aggregating useful information during the conversation. Therefore, sequence-to-sequence models such as the encoder-decoder based neural network models are proven to be suitable for both task-oriented and non-task-oriented systems. Serban et al. proposed to build end-to-end dialogue systems using generative hierarchical recurrent encoder-decoder neural network [2]. Li et al. presented persona-based models which incorporate background information and speaking style of interlocutors into LSTM-based seq2seq network so as to improve the modeling of human-like behavior [3]. Wen et al. designed an end-to-end trainable neural dialogue model with modularly connected components [4]. Bordes et al. [5] proposed a taskoriented dialogue model using end-to-end memory networks. At the same time, many works explored different kinds of networks to model the dialogue state, such as copy-augmented networks [6], gated memory networks [7], query-regression networks [8]. These systems do not perform slot-filling or user goal tracking; they rank and select a response from a set of response candidates which are conditioned on the dialogue history.

One of the significant effort in developing end-to-end taskoriented systems is the recent Sequicity framework [9]. This framework also relies on the sequence-to-sequence model and can be optimized with supervised or reinforcement learning. The Sequicity framework introduces the concept of belief span (bspan), which is a text span that tracks the dialogue states at each turn. In this framework, the task-oriented dialogue problem is decomposed into two stages: bspan generation and response generation. This framework has been shown to significantly outperform state-of-the-art pipeline-based methods.

The second line of work in SDS that is related to this work is concerned with multi-domain dialogue systems. As presented above, one of the key components of a dialogue system is dialogue state tracking, or belief tracking, which maintains the states of conversation. A state is usually composed of user's goals, evidences and information which is accumulated along the sequence of dialogue turns. While the user's goal and evidences are extracted from user's utterances, the useful information is usually aggregated from external resources such as knowledge bases or dialogue ontologies. Such knowledge bases contain slot type and slot value entries in one or several predefined domains. Most approaches have difficulty scaling up with multiple domains due to the dependency of their model parameters on the underlying knowledge bases. Recently, Ramadan et al. [10] has introduced a novel approach which 


\begin{tabular}{|c|c|}
\hline Turn & Dialogue \\
\hline$H_{1}$ & are there any chinese restaurants nearby? \\
\hline Match & $<$ inf $>$ chinese $</$ inf $><$ Req $><$ Rep $>$ \\
\hline$M_{1}$ & $\begin{array}{l}\text { the closest is POI_SLOT restaurant, and POI_SLOT is } \\
\text { DISTANCE_SLOT away with TRAFFIC_INFO }\end{array}$ \\
\hline $\mathrm{H}_{2}$ & what's the weather like today? \\
\hline Match & $\begin{array}{l}<\text { inf }>\text { chinese }</ \text { inf }\rangle<\text { inf }>\text { weather }</ \text { inf }> \\
<\langle\text { Req }></ \text { Rep }>\end{array}$ \\
\hline$M_{2}$ & what city are you wanting to know the weather for? \\
\hline $\mathrm{H}_{3}$ & for Newyork city please? \\
\hline Match & $\begin{array}{l}<\text { inf }>\text { chinese }</ \text { inf }><\text { inf }>\text { weather }</ \text { inf }> \\
<\text { Req }>\text { Newyork city }<\text { Rep }>\end{array}$ \\
\hline$M_{3}$ & $\begin{array}{l}\text { Today is WEATHER_ATTRIBUTE_SLOT, } \\
\text { and WEATHER_ATTRIBUTE_SLOT. }\end{array}$ \\
\hline
\end{tabular}

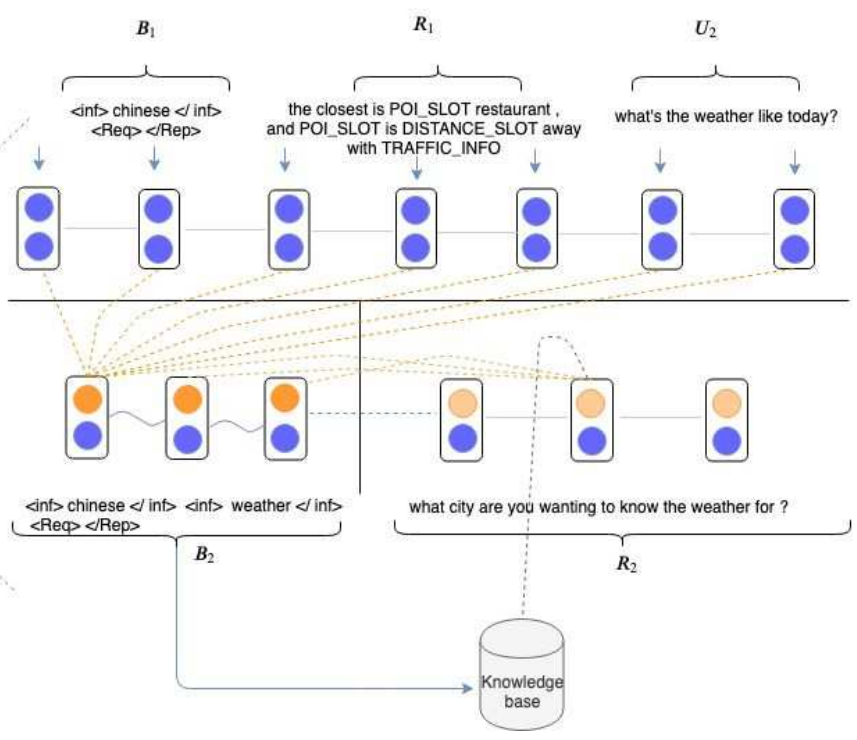

Fig. 1. Sequicity architecture.

utilizes semantic similarity between dialogue utterances and knowledge base terms, allowing the information to be shared across domains. This method has been shown not only to scale well to multi-domain dialogues, but also outperform existing state-of-the-art models in single-domain tracking tasks.

The problem that we are interested in this work is taskoriented dialogue in mixed-domain settings. This is different from the multi-domain dialogue problem above in several aspects, as follows:

- First, we investigate the phenomenon of alternating between different dialogue domains in subsequent dialogue turns, where each turn is defined as a pair of user question and machine answer. That is, the domains are mixed between turns. For example, in the first turn, the user requests some information of a restaurant; then in the second turn, he switches to the a different domain, for example, he asks about the weather at a specific location. In a next turn, he would either switch to a new domain or come back to ask about some other property of the suggested restaurant. This is a realistic scenario which usually happens in practical chatbot applications in our observations. We prefer calling this problem mixeddomain dialogue rather than multiple-domain dialogue.

- Second, we study the effect of the mixed-domain setting in the context of multi-domain dialogue approaches to see how they perform in different experimental scenarios.

The main findings of this work include:

- A specialized state tracking component in multiple domains still plays an important role and gives better results than a state-of-the-art end-to-end task-oriented dialogue system.

- A combination of specialized state tracking system and an end-to-end task-oriented dialogue system is beneficial in mix-domain dialogue systems. Our hybrid system is able to improve the belief tracking accuracy of about $28 \%$ of average absolute point on a standard multi-domain dialogue dataset.

- These experimental results give some useful insights on data preparation and acquisition in the development of the chatbot platform FPT.A1], which is currently deployed for many practical chatbot applications.

The remainder of this paper is structured as follows. First, Section [II discusses briefly the two methods in building dialogue systems that our method relies on. Next, Section [II presents experimental settings and results. Finally, Section IV concludes the paper and gives some directions for future work.

\section{Methodology}

In this section, we present briefly two methods that we use in our experiments which have been mentioned in the previous section. The first method is the Sequicity framework and the second one is the state-of-the-art multi-domain dialogue state tracking approach.

\section{A. Sequicity}

Figure 1 shows the architecture of the Sequicity framework as described in [9]. In essence, in each turn, the Sequicity model first takes a bspan $\left(B_{1}\right)$ and a response $\left(R_{1}\right)$ which are determined in the previous step, and the current human question $\left(U_{2}\right)$ to generate the current bspan. This bspan is then used together with a knowledge base to generate the corresponding machine answer $\left(R_{2}\right)$, as shown in the right part of Figure 1.

The left part of that figure shows an example dialogue in a mixed-domain setting (which will be explained in Section $\amalg$ ).

${ }^{1}$ http://fpt.ai/ 


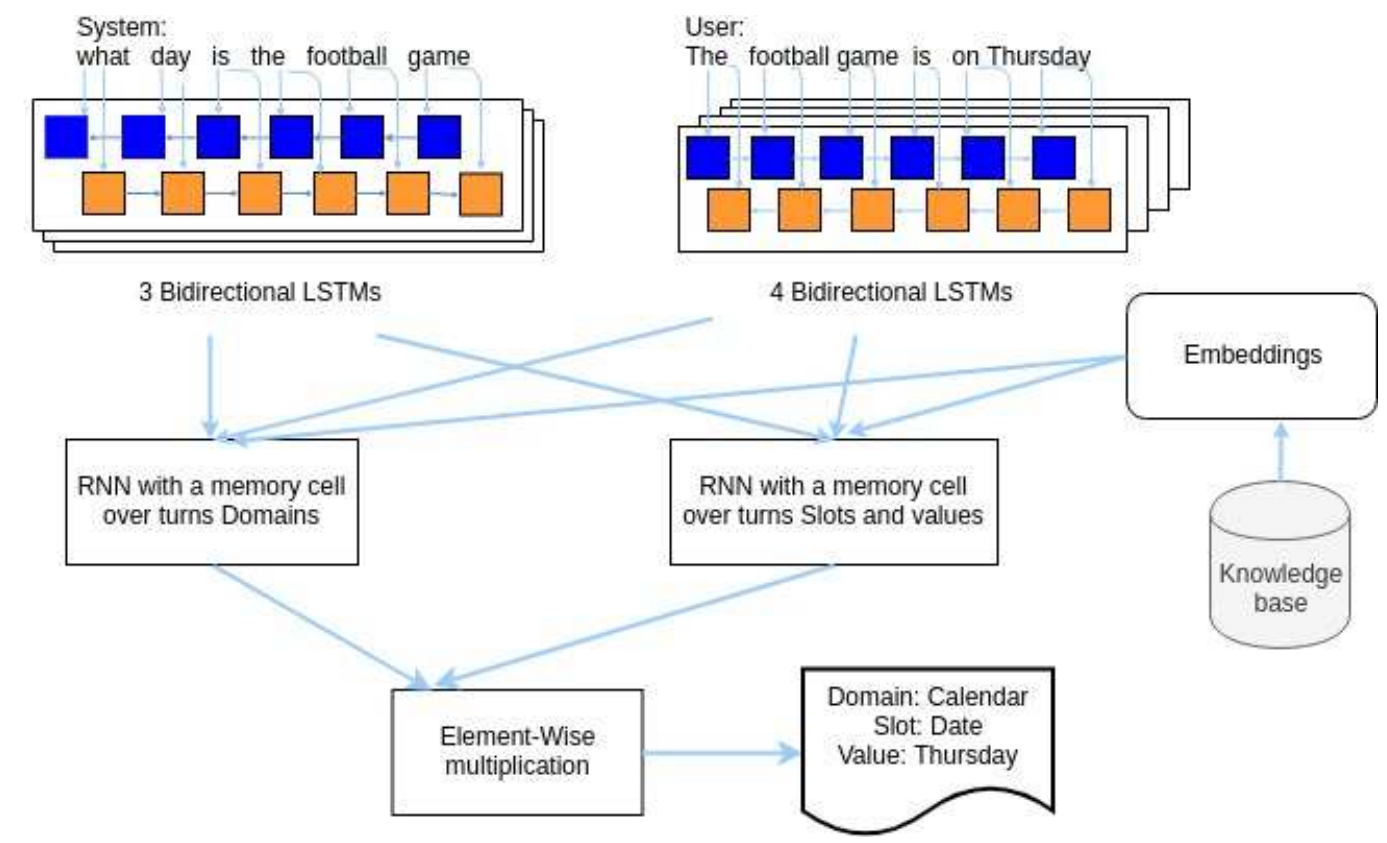

Fig. 2. Multi-domain belief tracking with knowledge sharing.

\section{B. Multi-domain Dialogue State Tracking}

Figure 2 shows the architecture of the multi-domain belief tracking with knowledge sharing as described in [10]. This is the state-of-the-art belief tracker for multi-domain dialogue.

This system encodes system responses with 3 bidirectional LSTM network and encodes user utterances with $3+1$ bidirectional LSTM network. There are in total 7 independent LSTMs. For tracking domain, slot and value, it uses 3 corresponding LSTMs, either for system response or user utterance. There is one special LSTM to track the user affirmation. The semantic similarity between the utterances and ontology terms are learned and shared between domains through their embeddings in the same semantic space.

\section{EXPERIMENTS}

In this section, we present experimental settings, different scenarios and results. We first present the datasets, then implementation settings, and finally obtained results.

\section{A. Datasets}

We use the publicly available dataset KVRET [6] in our experiments. This dataset is created by the Wizard-of-Oz method [11] on Amazon Mechanical Turk platform. This dataset includes dialogues in 3 domains: calendar, weather, navigation (POI) which is suitable for our mix-domain dialogue experiments. There are 2,425 dialogues for training, 302 for validation and 302 for testing, as shown in the upper half of Table 【

In this original dataset, each dialogue is of a single domain where all of its turns are on that domain. Each turn is composed of a sentence pair, one sentence is a user utterance, the other sentence is the corresponding machine response. A dialogue is a sequence of turns. To create mix-domain dialogues for our experiments, we make some changes in this dataset as follows:

- We keep the dialogues in the calendar domain as they are.

- We take a half of dialogues in the weather domain and a half of dialogues in the POI domain and mix their turns together, resulting in a dataset of mixed weatherPOI dialogues. In this mixed-domain dialogue, there is a turn in the weather domain, followed by a turn in POI domain or vice versa.

We call this dataset the sequential turn dataset. Since the start turn of a dialogue has a special role in triggering the learning systems, we decide to create another and different mixed-domain dataset with the following mixing method:

- The first turn and the last turn of each dialogue are kept as in their original.

- The internal turns are mixed randomly.

We call this dataset the random turn dataset. Some statistics of these mixed-domain datasets are shown in the lower half of the Table 【

\section{B. Experimental Settings}

For the task-oriented Sequicity model, we keep the best parameter settings as reported in the original framework, on the same KVRET dataset [9]. In particular, the hidden size of GRU unit is set to 50; the learning rate of Adam optimizer is 0.003 . In addition to the original GRU unit, we also re-run this framework with simple RNN unit to compare the performance of different recurrent network types. The Sequicity tool is freely available for download

\section{${ }^{2}$ https://github.com/WING-NUS/sequicity}


TABLE I

SOME STATISTICS OF THE DATASETS USED IN OUR EXPERIMENTS. THE ORIGINAL KVRET DATASET IS SHOWN IN THE UPPER HALF OF THE TABLE. THE MIXED DATASET IS SHOWN IN THE LOWER HALF OF THE TABLE.

\begin{tabular}{|c|c|c|c|c|}
\hline Dataset & \multicolumn{3}{|l|}{ KVRET } & \\
\hline Dialogues & \multicolumn{3}{|c|}{ Train: 2,425 ; Test: 302 ; Dev.: 302} & \\
\hline Domains & calendar & weather & POI & \\
\hline Dataset & \multicolumn{3}{|c|}{ MIXED DOMAINS } & \\
\hline Domains & calendar & weather & POI & Mixed weather - POI \\
\hline Train & 828 & 398 & 400 & 400 \\
\hline Test & 102 & 50 & 50 & 50 \\
\hline Dev. & 102 & 50 & 50 & 50 \\
\hline
\end{tabular}

For the multi-domain belief tracker model, we set the hidden size of LSTM units to 50 as in the original model; word embedding size is 300 and number of training epochs is 100 The corresponding tool is also freely available for download

\section{Results}

Our experimental results are shown in Table [II The first half of the table contains results for task-oriented dialogue with the Sequicity framework with two scenarios for training data preparation. For each experiment, we run our models for 3 times and their scores are averaged as the final score. The mixed training scenario performs the mixing of both the training data, development data and the test data as described in the previous subsection. The non-mixed training scenario performs the mixing only on the development and test data, keeps the training data unmixed as in the original KVRET dataset. As in the Sequicity framework, we report entity match rate, BLEU score and Success F1 score. Entity match rate evaluates task completion, it determines if a system can generate all correct constraints to search the indicated entities of the user. BLEU score evaluates the language quality of generated responses. Success F1 balances the recall and precision rates of slot answers. For further details on these metrics, please refer to [9].

In the first series of experiments, we evaluate the Sequicity framework on different mixing scenarios and different recurrent units (GRU or RNN), on two mixing methods (sequential turn or random turn), as described previously. We see that when the training data is kept unmixed, the match rates are better than those of the mixed training data. It is interesting to note that the GRU unit is much more sensitive with mixed data than the simple RNN unit with the corresponding absolute point drop of about $10 \%$, compared to about $3.5 \%$. However, the entity match rate is less important than the Success F1 score, where the GRU unit outperforms RNN in both sequential turn and random turn by a large margin. It is logical that if the test data are mixed but the training data are unmixed, we get lower scores than when both the training data and test data are mixed. The GRU unit is also better than the RNN unit on response generation in terms of BLEU scores.

We also see that the task-oriented dialogue system has difficulty running on mixed-domain dataset; it achieves only

\footnotetext{
${ }^{3}$ https://github.com/osmanio2/multi-domain-belief-tracking
}

about $75.62 \%$ of Success F1 in comparison to about $81.1 \%$ (as reported in the Sequicity paper, not shown in our table). Appendix A shows some example dialogues generated automatically by our implemented system.

In the second series of experiments, we evaluate the belief tracking components of two systems, the specialized multidomain belief tracker and the Sequicity bspan component. As shown in the lower half of the Table П. Sequicity capability of belief tracking is much worse than that of the multi-domain belief tracker. The slot accuracy gap between the tools is about $21.6 \%$, the value accuracy gap is about $34.4 \%$; that is a large average gap of $28 \%$ of accuracy. This result suggests a future work on combining a specialized belief tracking module with an end-to-end task-oriented dialogue system to improve further the performance of the overall dialogue system.

\section{Error Analysis}

In this subsection, we present an example of erroneous mixed dialogue with multple turns. Table $\amalg$ shows a dialogue in the test set where wrong generated responses of the Sequicity system are marked in bold font.

In the first turn, the system predicts incorrectly the bspan, thus generates wrong slot values (heavy traffic and Pizza Hut). The word Pizza Hut is an arbitrary value selected by the system when it cannot capture the correct value home in the bspan. In the second turn, the machine is not able to capture the value this_week. This failure does not manifest immediately at this turn but it is accumulated to make a wrong answer at the third turn (monday instead of this_week).

The third turn is of domain weather and the fourth turn is switched to domain POI. The bspan value cleveland is retained through cross domain, resulting in an error in the fourth turn, where cleveland is shown instead of home. This example demonstrates a weakness of the system when being trained on a mixed-domain dataset. In the fifth turn, since the system does not recognize the value fastest in the bspan, it generates a random and wrong value moderate traffic. Note that the generated answer of the sixth turn is correct despite of the wrong predicted bspan; however, it is likely that if the dialogue continues, this wrong bspan may result in more answer mistakes. In such situations, multi-domain belief tracker usually performs better at bspan prediction. 
TABLE II

Our eXperimental Results. Match. and SuCC. F1 are Entity match Rate and Success F1. The upper half OF the table Shows Results OF TASK-ORIENTED DIALOGUE WITH THE SEQUICITY FRAMEWORK. THE LOWER HALF OF THE TABLE SHOWS RESULTS OF MULTI-DOMAIN BELIEF

TRACKER.

\begin{tabular}{|c|c|c|c|c|c|c|c|}
\hline \multirow{2}{*}{ Sequicity } & & \multicolumn{3}{|c|}{ Case 1 - sequential turn } & \multicolumn{3}{|c|}{ Case 2 - random turn } \\
\hline & & Match. & BLEU & Succ. F1 & Match. & $\overline{B L E U}$ & Succ. F1 \\
\hline \multirow{2}{*}{ mixed training } & GRU & 0.6367 & $\overline{0.1930}$ & $\overline{0.7358}$ & 0.6860 & 0.1862 & 0.7562 \\
\hline & RNN & 0.7354 & 0.1847 & 0.7129 & 0.6591 & 0.1729 & 0.7105 \\
\hline \multirow[t]{2}{*}{ non-mixed training } & GRU & 0.7399 & 0.1709 & 0.7055 & $\overline{0.7488}$ & 0.1820 & 0.7173 \\
\hline & RNN & 0.7706 & 0.1453 & 0.6156 & 0.6995 & 0.1580 & 0.6633 \\
\hline & & $\begin{array}{l}\text { Domain - } \\
\text { accuracy }\end{array}$ & $\begin{array}{c}\text { Slot - } \\
\text { accuracy }\end{array}$ & $\begin{array}{c}\text { Value - } \\
\text { accuracy }\end{array}$ & & & \\
\hline \multirow[t]{2}{*}{ Belief tracker } & Multi-domain & 0.8253 & 0.9329 & 0.9081 & & & \\
\hline & Sequicity & & 0.7171 & 0.5644 & & & \\
\hline
\end{tabular}

TABLE III

A MIXED DIALOGUE EXAMPLE IN THE TEST SET WITH ERRONEOUS GENERATED RESPONSES. THE LAST TWO COLUMNS SHOW RESPECTIVELY THE SYSTEM'S GENERATED BSPAN AND THE GOLD BSPAN OR BELIEF TRACKER.

\begin{tabular}{|l|l|l|l|l|}
\hline Turn & Dialogue & Domain & Generated bspan & Gold bspan \\
\hline 1.Human & car please send me the fastest route to go back home & POI & EOS_Z1 & home fastest EOS_Z1 \\
\hline 1.Machine & there is heavy traffic on the way to Pizza Hut. & POI & & \\
\hline 2.Human & what is the weather like this week & Weather info & EOS_Z1 & this_week EOS_Z1 \\
\hline 2.Machine & what city are you interested in ? & Weather info & & \\
\hline 3.Human & Cleveland . & Weather info & cleveland EOS_Z1 & cleveland EOS_Z1 \\
\hline 3.Machine & it will be clear sky on monday & Weather info & & \\
\hline 4.Human & what is the address ? & POI & cleveland EOS_Z1 & home fastest EOS_Z1 \\
\hline 4.Machine & cleveland is located at 5672 barringer street & POI & & \\
\hline 5.Human & $\begin{array}{l}\text { please send me the fastest route on screen and let 's go there } \\
\text { thank you ! }\end{array}$ & POI & cleveland EOS_Z1 & home fastest EOS_Z1 \\
\hline 5.Machine & $\begin{array}{l}\text { there is moderate traffic on our way but i sent the route to } \\
\text { your screen , drive carefully ! }\end{array}$ & POI & & \\
\hline 6.Human & thank & & cleveland EOS_Z1 & EOS_Z1 \\
\hline 6.Machine & you are welcome . & & & \\
\hline
\end{tabular}

\section{CONCLUSION}

We have presented the problem of mixed-domain taskoriented dialogue and its empirical results on two datasets. We employ two state-of-the-art, publicly available tools, one is the Sequicity framework for task-oriented dialogue, and another is the multi-domain belief tracking system. The belief tracking capability of the specialized system is much better than that of the end-to-end system. We also show the difficulty of task-oriented dialogue systems on mixed-domain datasets through two series of experiments. These results give some useful insights in combining the approaches to improve the performance of a commercial chatbot platform which is under active development in our company. We plan to extend this current research and integrate its fruitful results into a future version of the platform.

\section{REFERENCES}

[1] Bing Liu, Gokhan Tur, Dilek Hakkani-Tur, Pararth Shah, and Larry Heck. Dialogue learning with human teaching and feedback in end-toend trainable task-oriented dialogue systems. In Proceedings of NAACL, 2018.

[2] Iulian Serban, Alessandro Sordoni, Yoshua Bengio, Aaron C. Courville, and Joelle Pineau. Building end-to-end dialogue systems using generative hierarchical neural network models. In Proceedings of AAAI, 2016.

[3] Jiwei Li, Michel Galley, Chris Brockett, Georgios P. Spithourakis, Jianfeng Gao, and Bill Dolan. A persona-based neural conversation model. In Proceedings of ACL, 2016.
[4] Tsung-Hsien Wen, David Vandyke, Nikola Mrksic, Milica Gasic, Lina M. Rojas-Barahona, Pei-Hao Su, Stefan Ultes, and Steve Young. A network-based end-to-end trainable task-oriented dialogue system. In Proceedings of EACL, 2017.

[5] Antoine Bordes, Y-Lan Boureau, and Jason Weston. Learning end-toend goal-oriented dialogue. In Proceedings of ICLR, 2017.

[6] Mihail Eric and Christopher D. Manning. A copy-augmented sequenceto-sequence architecture gives good performance on task-oriented dialogue. In Proceedings of EACL, 2017.

[7] Fei Liu and Julien Perez. Gated end-to-end memory networks. In Proceedings of EACL, 2017.

[8] Min Joon Seo, Hannaneh Hajishirzi, and Ali Farhadi. Query-regression networks for machine comprehension. In Preprint ArXiv, 2016.

[9] Wenqiang Lei, Xisen Jin, Zhaochun Ren, Xiangnan He, Min-Yen Kan, and Dawei Yin. Sequicity: Simplifying task-oriented dialogue systems with single sequence-to-sequence architectures. In Proceedings of $A C L$, 2018.

[10] Milica Gasic Osman Ramadan, Paweł Budzianowski. Large-scale multidomain belief tracking with knowledge sharing. In Proceedings of ACL, 2018.

[11] John F Kelley. iterative design methodology for user-friendly natural language office information applications. ACM Transactions on Information Systems, 2(1):26-41, 1984. 


\section{APPENDIX}

The following is three example dialogues generated by our system. The first dialogue is in single-domain.

\begin{tabular}{|c|c|c|}
\hline Turn & Dialogue 1 & Domain \\
\hline Human & $\begin{array}{l}\text { What time is my next din- } \\
\text { ner event and who will be } \\
\text { attending? }\end{array}$ & Calendar \\
\hline Machine & $\begin{array}{l}\text { your dinner is on MONDAY } \\
\text { at } 5 \mathrm{PM} \text {. }\end{array}$ & Calendar \\
\hline Human & That will do, thanks. & \\
\hline Machine & you are welcome & \\
\hline
\end{tabular}

The next two dialogues are in mixed-domains.

\begin{tabular}{|c|c|c|}
\hline Turn & Dialogue 2 & Domain \\
\hline Human & $\begin{array}{l}\text { Please give me the ad- } \\
\text { dress and directions to home } \\
\text { within } 5 \text { miles of here, } \\
\text { avoiding all heavy traffic. }\end{array}$ & POI \\
\hline Machine & $\begin{array}{l}\text { HOME is located at } 5671 \\
\text { BARRINGER STREET }\end{array}$ & POI \\
\hline Human & $\begin{array}{l}\text { what is the forecast for the } \\
\text { next } 7 \text { days? }\end{array}$ & weather info \\
\hline Machine & $\begin{array}{l}\text { what city are you interested } \\
\text { in ? }\end{array}$ & weather info \\
\hline Human & Cleveland, will it be windy? & weather info \\
\hline Machine & $\begin{array}{l}\text { it will not be windy in } \\
\text { Cleveland this week. }\end{array}$ & weather info \\
\hline Human & Thank you. & \\
\hline Machine & you're welcome. & \\
\hline Turn & Dialogue 3 & Domain \\
\hline Human & $\begin{array}{l}\text { are there any chinese restau- } \\
\text { rants nearby? }\end{array}$ & POI \\
\hline Machine & $\begin{array}{l}\text { the closest is TAI PAN } \\
\text { restaurant, and TAI PAN } \\
\text { is } 5 \text { miles away with NO } \\
\text { TRAFFIC }\end{array}$ & POI \\
\hline Human & $\begin{array}{l}\text { what's the weather like to- } \\
\text { day? }\end{array}$ & weather info \\
\hline Machine & $\begin{array}{l}\text { what city are you wanting to } \\
\text { know the weather for? }\end{array}$ & weather info \\
\hline Human & $\begin{array}{lcc}\text { for } & \text { Newyork } & \text { city } \\
\text { please?weather info } & \end{array}$ & \\
\hline Machine & $\begin{array}{l}\text { Today is CLEAR SKIES, } \\
\text { and WARM. }\end{array}$ & weather info \\
\hline Human & $\begin{array}{l}\text { ok, good, set the direction to } \\
\text { there }\end{array}$ & POI \\
\hline Machine & $\begin{array}{l}\text { the address is } 830 \text { Almanor } \\
\text { Ln, i sent it on your screen }\end{array}$ & POI \\
\hline Human & ok thanks & \\
\hline Machine & you're welcome! & \\
\hline
\end{tabular}

